\title{
VISUALIZATION OF DATA FROM NETWORK OF SENSORS: APPROPRIATE SPATIAL INTERPOLATION METHOD
}

\author{
Adnan Masic, Dzevad Bibic, Boran Pikula, Emina Dzaferovic-Masic \& Faruk Razic
}
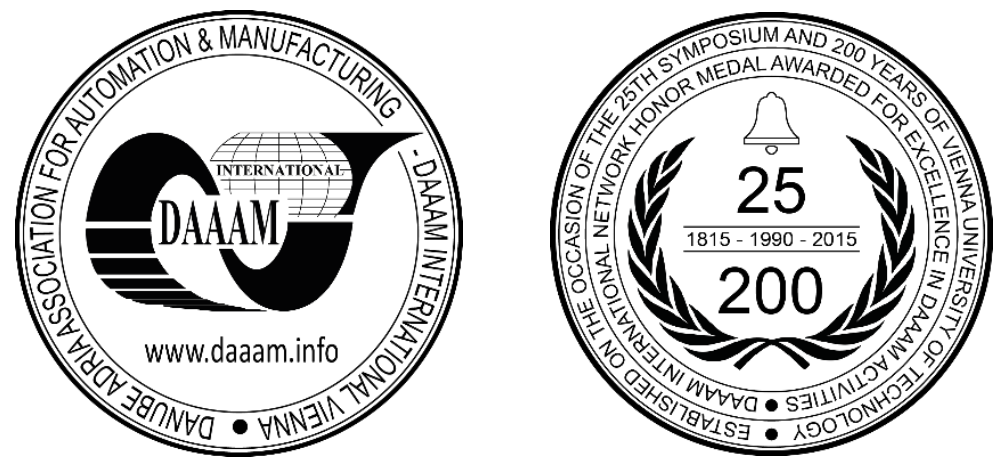

This Publication has to be referred as: Masic, A[dnan]; Bibic, D[zevad]; Pikula, B[oran]; Dzaferovic-Masic, E[mina] \& Razic, F[aruk] (2018). Visualization of Data from Network of Sensors: Appropriate Spatial Interpolation Method, Proceedings of the 29th DAAAM International Symposium, pp.0529-0533, B. Katalinic (Ed.), Published by DAAAM International, ISBN 978-3-902734-20-4, ISSN 1726-9679, Vienna, Austria

DOI: $10.2507 / 29$ th.daaam.proceedings.076

\begin{abstract}
Graphical presentation of the measured data is more popular than ever before. However, rich and colourful images are often misrepresentation of the measured quantities. In this paper we discuss proper mathematical technique for correct presentation of data from the network of sensors. Three common methods for spatial interpolation are demonstrated and compared using real data from the network of sensors (for air pollution with aerosols). Finally, best procedure is recommended and discussed in details.
\end{abstract}

Keywords: network of sensors; spatial interpolation; Hilbert space; air pollution

\section{Introduction}

Number of sensors of various types that are connected to the internet is increasing rapidly. This fits into trends popularly called "Internet of Things" and "Smart Cities". Despite the fact that number of sensors is increasing, we will never have a sensor at every single point of interest. Therefore, some sort of spatial interpolation is needed. The main question is: if we have $N$ sensors over certain geographical area, which spatial interpolation method is appropriate for visualization of measured quantity over the entire area? In this paper we will try to answer that question using the real data from the network of sensors that measure air pollution.

The simplest approach is to use bilinear interpolation [1]:

$f(x, y)=a_{0}+a_{1} x+a_{2} y+a_{3} x y$

where $a_{0}, a_{1}, a_{2}$ and $a_{3}$ are coefficients which can be calculated by knowing values of function $f$ at four different points around (four sensors from the network). Obviously, this is not the best method, due to the nature of sensors (inevitable errors during the measurements) and local disturbances (very localized source of air pollution for example). 
More natural approach is inversed distance weighting [2]. This is a multivariate interpolation with a known scattered set of points. The assigned values to unknown points are calculated with a weighted average of the values available at the known points. This method gives few options of choosing parameters, so that the nature of the measurements is represented properly.

Next candidate is the procedure called kriging [3] (name given from the master thesis of Danie Krige [4]). Kriging is a method of interpolation for which the interpolated values are modelled by a Gaussian process governed by prior covariance. The kriging estimation may also be seen as a (sort of) spline in a Hilbert space. When performing kriging, the user must specify the function which describes the degree of spatial dependence of a spatial random field - this is called variogram. Under suitable assumptions, kriging gives the best linear unbiased prediction of the intermediate values [5].

\section{Case study}

Network of 13 sensors which measure the concentration of aerosols smaller than $10 \mu \mathrm{m}$ (PM10) was installed in and around the city of Sarajevo. Daily average values of PM10 for 30/08/2018 are chosen for this study. Figure 1 illustrates the location of sensors, while table 1 shows calculated average values of PM10 for each sensor. The method for data acquisition is explained in [6] and [7], while the calibration of PM10 sensor was described in [8].

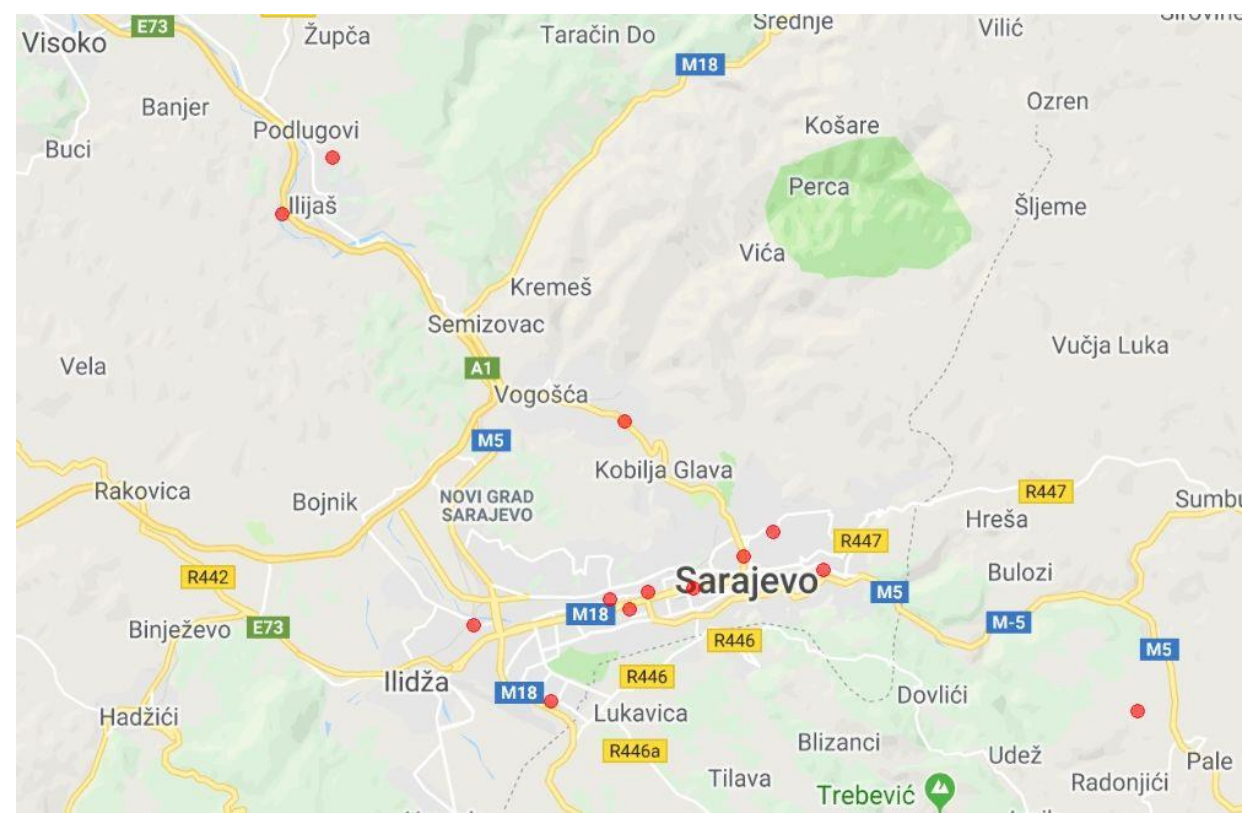

Fig. 1. Location of sensors

\begin{tabular}{|c|c|c|c|}
\hline sensor & latitude & longitude & PM10 $\left(\mu \mathrm{g} / \mathrm{m}^{3}\right)$ \\
\hline 1 & 43.89488 & 18.37228 & 33.51 \\
\hline 2 & 43.82361 & 18.54752 & 26.09 \\
\hline 3 & 43.86172 & 18.41277 & 34.47 \\
\hline 4 & 43.84860 & 18.37393 & 39.53 \\
\hline 5 & 43.85390 & 18.39553 & 37.80 \\
\hline 6 & 43.85284 & 18.38026 & 41.12 \\
\hline 7 & 43.84482 & 18.32074 & 41.66 \\
\hline 8 & 43.85846 & 18.44020 & 32.04 \\
\hline 9 & 43.86775 & 18.42296 & 33.27 \\
\hline 10 & 43.95983 & 18.27251 & 47.09 \\
\hline 11 & 43.94594 & 18.25530 & 46.14 \\
\hline 12 & 43.85121 & 18.36727 & 41.40 \\
\hline 13 & 43.82605 & 18.34702 & 40.01 \\
\hline
\end{tabular}

Table 1. Daily average values of PM10 for 30/8/2018 


\section{Spatial interpolation methods}

Bilinear interpolation was applied in figure 2. Akima library for R programming language was used for calculations of interpolated values. Contour lines (for values of PM10) were added, together with locations of sensors. There is no interpolation outside the polygon of sensors. We can also see that this spatial interpolation of PM10 doesn't look natural because values from sensors around the city linearly propagate all the way to the city center.

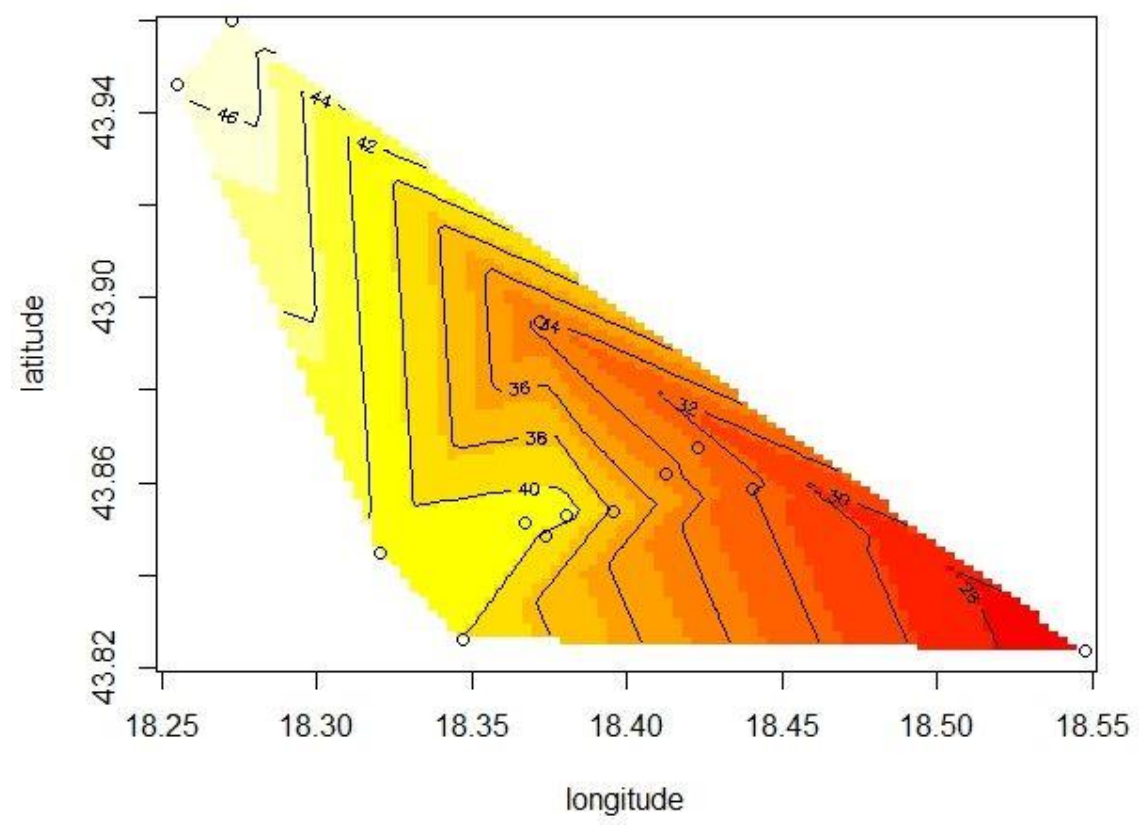

Fig. 2. Bilinear interpolation

Figure 3 shows inverse distance weighting (IDW) interpolation with three different power factors $(1,2$ and 3 ). Calculations were performed in $\mathrm{R}$ programming language again. Contour lines look more natural now. However, we don't know a priori which power factor is correct.

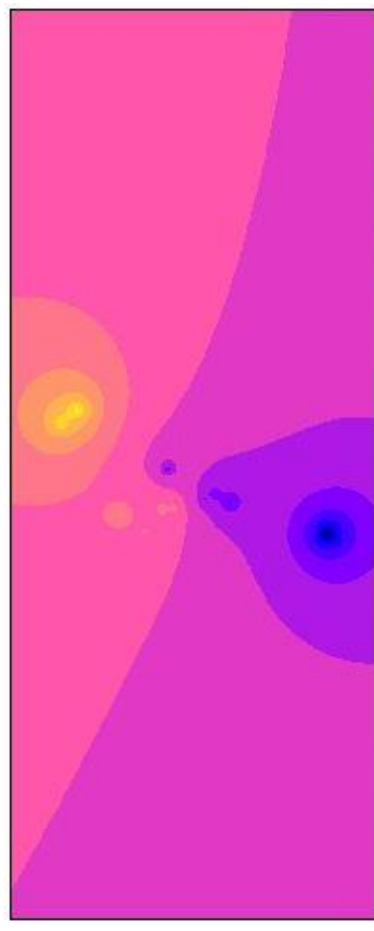

$p=1$

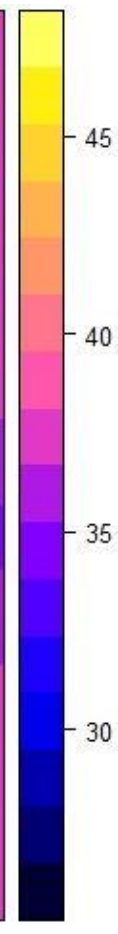

$-30$

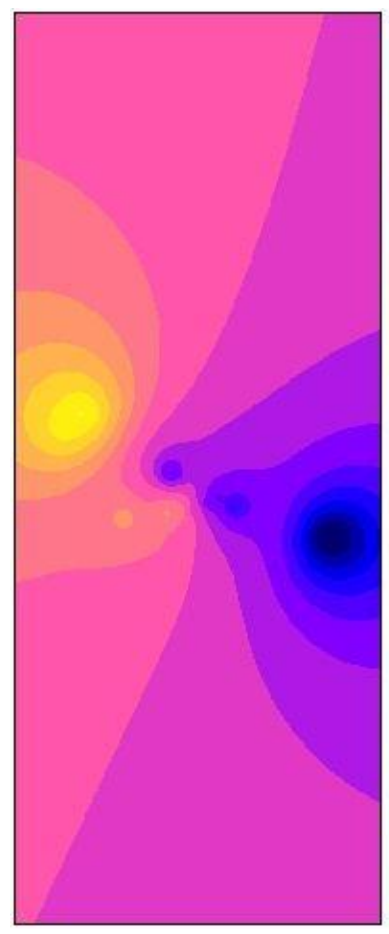

$p=2$

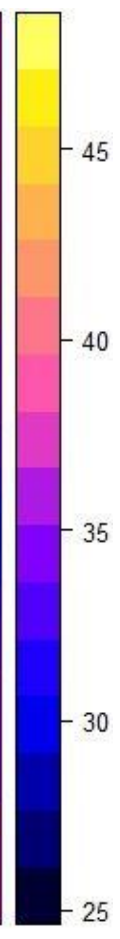

25

.

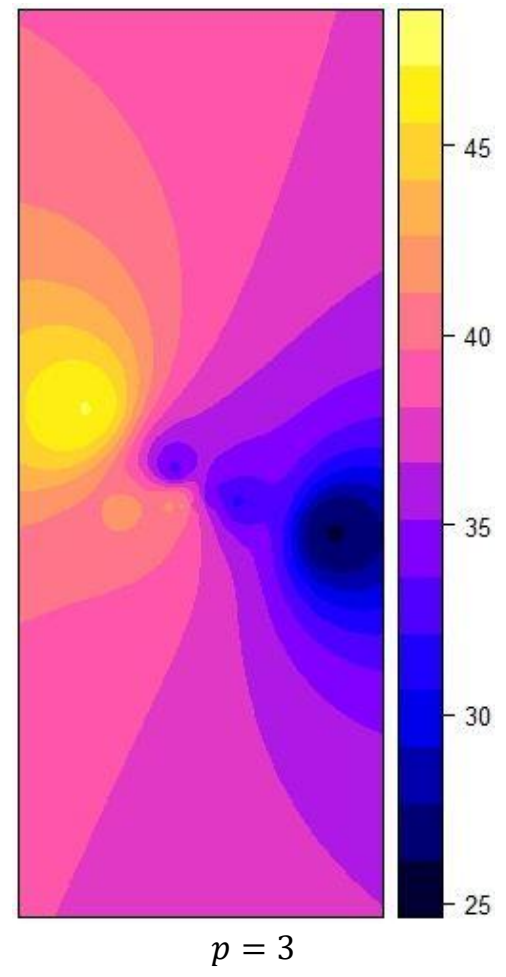

Fig. 3. Inverse distance weighting interpolation with three different power coefficients 
Now we proceed to the kriging. As noted above, first thing we need to do is to choose the proper variogram. Figure 4 shows the dependence of semivariance on distance, for the selected dataset. We can see from the graph that the Gaussian fit is the best option. Using the Gaussian fit from figure 4 we can finally perform kriging interpolation. Figure 5 shows the result of this operation. We can also estimate quality of interpolation by means of cross-validation: it removes each data location, one at a time and predicts the associated data value. R programming language was used for plotting kriging interpolation and cross-validation errors in figure 5. We would like to note that IDW and kriging techniques can be used to extrapolate data as well. Please note the location of sensors in figure 1: we can't have good estimation in the region where we don't have sensors at all!

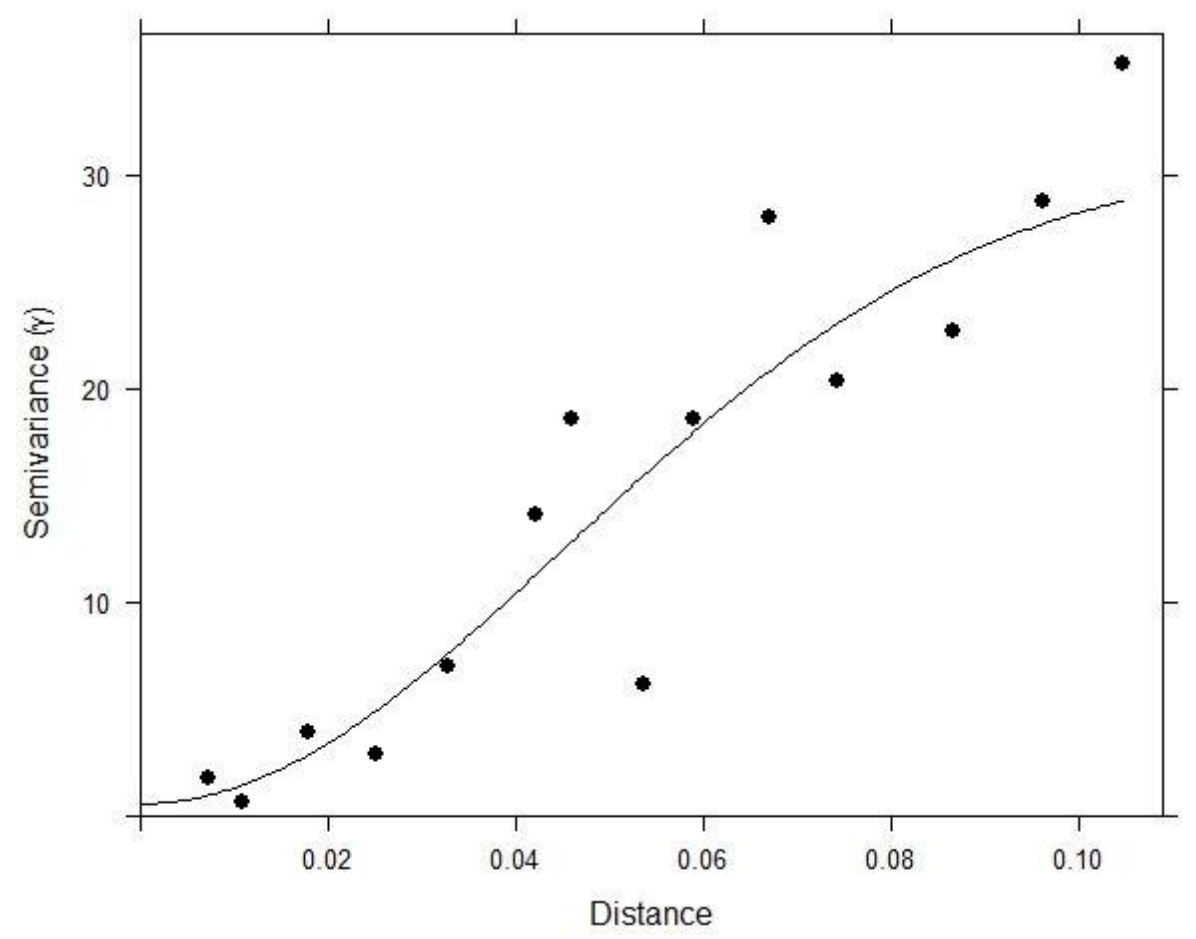

Fig. 4. Variogram and Gaussian fit

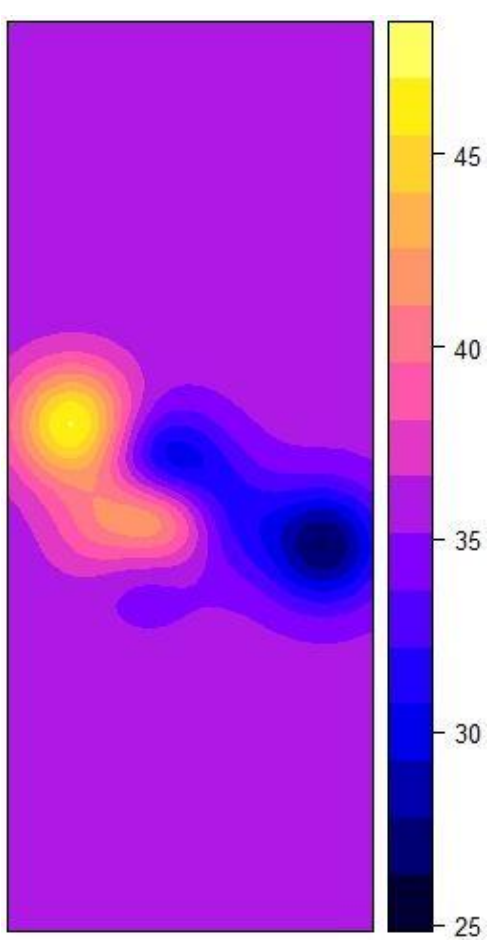

kriging interpolation

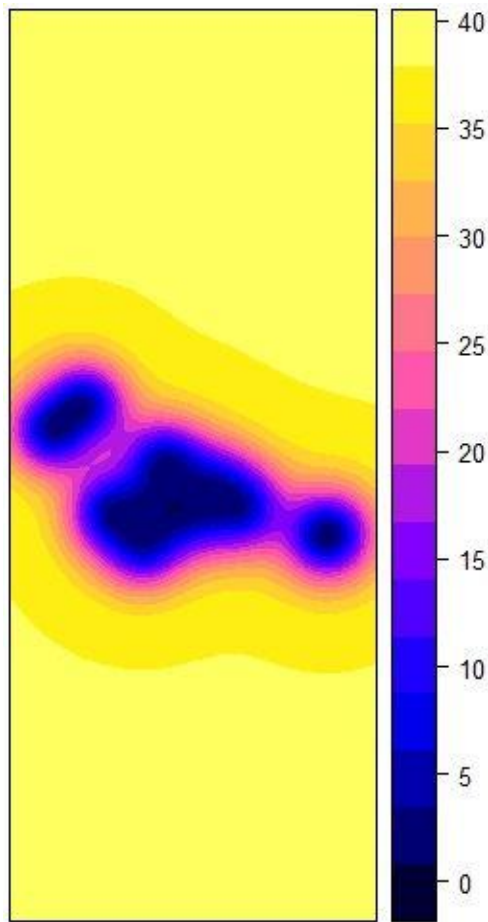

cross-validation errors

Fig. 5. Kriging interpolation and cross-validation 


\section{Conclusion}

Three different techniques for interpolation of data from network of sensors were analysed: bilinear, IDW and kriging interpolation. They produced significantly different contour lines of PM10 concentrations from the same dataset. Bilinear interpolation should not be used for such cases (where we have non-trivial spatial correlation of data). IDW interpolation gives more natural contour lines, but it requires parameters that we don't know a priori. The final conclusion is very clear: kriging is the preferable method. If the variogram fit is chosen properly, kriging is the best interpolation technique among these three (and probably best of all, for most general applications).

As a suggestion for further research we propose:

- Extension of network of sensors to more different places,

- Measurement of various episodes of air pollution, especially during the winter,

- Analysis of more variables such as altitude, humidity etc.

\section{Acknowledgments}

This research was supported by the Ministry of Education of Sarajevo Canton and the University of Sarajevo Faculty of Mechanical Engineering. We would like to thank to Mr. Damir Muslić for his generous contribution to our project by writing core part of the in-house developed software.

\section{References}

[1] Press, W. H.; Flannery, B. P.; Teukolsky, S. A. \& Vetterling, W. T. (1992). Numerical Recipes in C: The Art of Scientific Computing, Second Edition. Cambridge University Press. ISBN-13: 978-0521431088, New York

[2] Łukaszyk, S. (2004). A New Concept of Probability Metric and its Applications in Approximation of Scattered Data Sets. Computational Mechanics, 33 (2004) 299-304, DOI: 10.1007/s00466-003-0532-2

[3] Cressie, N. A. C. (1990). The Origins of Kriging, Mathematical Geology, 22 (1990) 239-252

[4] Krige, D. G. (1951). A Statistical Approach to Some Mine Valuations and Allied Problems at the Witwatersrand, Master's thesis, University of Witwatersrand, South Africa

[5] Oliver, M. A. (1990). Kriging: A Method of Interpolation for Geographical Information Systems. International Journal of Geographic Information Systems, 4 (1990) 313-332

[6] Masic, A.; Pikula, B. \& Bibic, D. (2017). Mobile Measurements of Particulate Matter Concentrations in Urban Area, Proceedings of the 28th DAAAM International Symposium, pp.0452-0456, B. Katalinic (Ed.), Published by DAAAM International, ISBN 978-3-902734-11-2, ISSN 1726-9679, Vienna, Austria DOI: 10.2507/28th.daaam.proceedings.063

[7] Masic, A.; Bibic, D.; Pikula, B.; Dzaferovic-Masic, E. \& Musemic, R. (2018). Experimental Study of Temperature Inversions Above Urban Area Using Unmanned Aerial Vehicle, Thermal Science (2018)

[8] Masic, A.; Pikula, B.; Bibic, D.; Musemic, R. \& Halac, A. (2018). Calibration and Assessment of Low-cost Dust sensors, Proceedings of the 29th DAAAM International Symposium, B. Katalinic (Ed.), Published by DAAAM International, ISSN 1726-9679, Vienna, Austria, in press 